\title{
РЕАЛИЗАЦИЯ КОМПЛЕКСНОЙ ЦИФРОВОЙ СИСТЕМЫ ФАПЧ ДЛЯ ФАЗОВОГО ДЕТЕКТИРОВАНИЯ В ПРОГРАММНО ОПРЕДЕЛЯЕМОМ РАДАРЕ
}

\author{
МАНДАЛ А., МИШРА Р., НАГАР М. Р. \\ Университет Гаутамма Будды, \\ Индия, Большая Нойда, 201308
}

\begin{abstract}
Аннотация. Программно определяемый радар SDR (software defined radar) представляет собой новейшее направление в разработке расширенных методик обработки сигнала радара для современных радиолокационных систем. SDR обеспечивает высокую гибкость перестройки структуры и быстрое макетирование на платформе FPGA (программируемая логическая интегральная схема, ПЛИС). Для осуществления обработки данных в реальном времени быстродействующего радара используется цифровой компьютер координационного вращения CORDIC (COordinate Rotation Digital Computer) в качестве основного процессорного элемента в комплексной цифровой системе фазовой автоподстройки частоты (ФАПЧ) для цифровой демодуляции принятого сигнала. Поскольку системы реального времени работают с высокими частотами дискретизации, выбрана конвейерная архитектура процессорного элемента CORDIC для обеспечения высокой скорости обработки (пропускной способности). Эта архитектура оптимизирована в отношении битовой длины для лучшей сходимости и повышения качества работы контура комплексной ФАПЧ первого порядка во время демодуляции. Узкополосный фильтр типа BOXCAR использован в качестве фильтра нижних частот в выходном каскаде детектора для улучшения извлечения информации из узких выборок при малой энергии сигнала без необходимости дополнительных затрат аппаратных средств. Приведены результаты моделирования в среде MATLAB для демонстрации эффективности этой конструкции интегральной схемы применительно к фазовому детектированию в радаре
\end{abstract}

Ключевые слова: программно определяемый радар; SDR; цифровая фазовая автоподстройка частоты; ФАПЧ; алгоритм CORDIC; фильтр BOXCAR; демодуляция

\section{1. ВСТУПЛЕНИЕ}

Программно определяемый радар SDR (software defined radar) представляет собой наиболее универсальную систему, в которой большая часть аппаратной обработки заменена программными средствами при сохранении высокой точности. SDR обладает уникальной способностью получать высокоточную информацию, касающуюся скорости движущегося объекта, дальности и направления $[1,2]$. Фазовое детектирование в приемнике радара (РЛС) представляет собой важный и критичный про- цесс при обнаружении цели в реальном времени.

Управляемый напряжением генератор (УНГ) используется во многих системах радиосвязи, включая радарные системы. Однако характерная для такого генератора нелинейность и отсутствие в нем спектральной чистоты затрудняет процесс фазового детектирования для получения спектрально чистого выходного сигнала в необходимом диапазоне частот $[3,4]$. Цифровые синтезаторы частоты прямого действия DDFS (direct digital frequency synthesizer), основанные на модуляторах/демодуляторах, широко применяются в 